\title{
Dengue virus type 3 in Brazil: a phylogenetic perspective
}

\author{
Josélio Maria Galvão de Araújo ${ }^{1 /+}$, Gonzalo Bello², Hermann Gonçalves Schatzmayr ${ }^{1}$, \\ Flávia Barreto dos Santos ${ }^{1}$, Rita Maria Ribeiro Nogueira ${ }^{1}$
}

'Laboratório de Flavivirus ²aboratório de AIDS e Imunologia Molecular, Instituto Oswaldo Cruz-Fiocruz, Av. Brasil 4365, 21045-900 Rio de Janeiro, RJ, Brasil

Circulation of a new dengue virus (DENV)-3 genotype was recently described in Brazil and Colombia, but the precise classification of this genotype has been controversial. Here we perform phylogenetic and nucleotidedistance analyses of the envelope gene, which support the subdivision of DENV-3 strains into five distinct genotypes (GI to GV) and confirm the classification of the new South American genotype as GV. The extremely low genetic distances between Brazilian GV strains and the prototype Philippines/L11423 GV strain isolated in 1956 raise important questions regarding the origin of GV in South America.

Key words: dengue virus type 3 - genotypes - Brazil

Dengue viruses (DENV) 1-4, members of the Flavivirus genus within the Flaviviridae family, are currently responsible for more deaths worldwide than any other arthropod-transmitted human viral disease, and are emerging as a major problem in tropical and subtropical areas (Rosen 1999). The $\sim 11 \mathrm{~kb}$-long genomic RNA of DENV is a single strand of positive polarity, comprised of a single open reading frame (ORF) flanked by untranslated regions at the $5^{\prime}$ and $3^{\prime}$ termini. The ORF codes for three structural proteins [capsid (C), premembrane (prM/M) and envelope (E)] and seven nonstructural proteins (NS1, NS2A, NS2B, NS3, NS4A, NS4B and NS5), resulting from the cleavage of a single polyprotein of 3386-3433 amino acids (Chambers et al. 1990, Lindebach \& Rice 2001).

A phylogenetic analysis of the $\mathrm{prM} / \mathrm{M}$ and $\mathrm{E}$ genes of the geographically and temporally-distinct DENV-3 led to the initial identification of four distinct monophylogenetic clusters called genotypes (Lanciotti et al. 1994). A subsequent phylogenetic analysis of a larger number of DENV-3 strains demonstrated that genotype I of Lanciotti et al. (1994) encompasses two distinct groups of viruses that were previously classified separately, leading to the establishment of a new genotype (V). Genotypes I-III (GI-GIII) are responsible for most DENV-3 infections: GI is represented by viruses from Indonesia, Malaysia, Philippines and the South Pacific; GII by viruses from Thailand, Bangladesh, Malaysia and Myanmar; and GIII by viruses from Sri Lanka, India, Samoa, Africa and the Americas (sampled after 1994). In contrast, genotypes IV and V (GIV and GV) are only found in a

Financial support: CNPq (501564/03-9), FAPERJ (E-26/152490/2002) JMG Araújo and G Bello contributed equally to this article. + Corresponding author: joselio@ioc.fiocruz.br

Received 7 August 2008

Accepted 3 March 2009 few older strains: GIV in Puerto Rican strains from the 1960s/1970s and GV in the oldest strain isolated in the Philippines in 1956, as well as in two strains isolated in Japan and China in 1973 and 1980, respectively.

DENV-3 GIII was first detected in the Americas during dengue fever/dengue hemorrhagic fever (DF/DHF) outbreaks in Nicaragua and Panama, in 1994 (Anonymous 1995). Subsequently, this virus has spread to many countries in Latin America (Briseno-Garcia et al. 1996, Guzman et al. 1998, Balmaseda et al. 1999, Istúriz et al. 2000, Nogueira et al. 2001, Usuku et al. 2001, Uzcategui et al. 2003, Aquino et al. 2006). In Brazil, DENV-3 was first isolated from an autochthonous case of DF in December 2000 from the municipality of Nova Iguaçu, state of Rio de Janeiro, and later there was a large DENV-3 epidemic in Rio de Janeiro in 2001/2002 (Nogueira et al. 2001). These DENV-3 isolates appeared to have arisen from a single introduction of GIII strains previously circulating in Sri Lanka, India and East Africa, probably around 1991 (1987-1993) (Araujo et al. 2009). However, a new DENV-3 genotype has recently been reported to be circulating in Brazil (Figueiredo et al. 2008, Nogueira et al. 2008) and Colombia (Usme-Ciro et al. 2008) and was classified as GI by Figueiredo et al. (2008) and Usme-Ciro et al. (2008), but as GV by Nogueira et al. (2008). The different classifications clearly indicate the need for an international standardisation of the DENV-3 genotype nomenclature. For this reason, we re-examined the current phylogenetic classification of DENV-3 strains with emphasis on the new genotype described in Brazil.

We retrieved (Genbank, www.ncbi.nlm.nih.gov) and analyzed the full-length $(1,479 \mathrm{bp})$ and partial (822 bp) E gene sequences of 103 DENV-3 strains from 30 different countries around the world, representative of all known genotypes. Colombian sequences described by Usme-Ciro et al. (2008) were not included in this study, as only a short E gene fragment (224 bp) was available. Tree reconstructions were performed by the NeighbourJoining method (Tamura 2007) in 1,000 bootstrapped data sets, as implemented in MEGA 4. Mean nucleotide distances within (intra-genotype) and among (inter- 
genotype) DENV-3 genotypes, and pair-wise genetic distances among sequences, were estimated by adopting the Tamura Nei model in MEGA 4.

Our phylogenetic analysis of DENV-3 E gene sequences confirmed the five monophyletic groups (geno- types) previously reported for this serotype (Figure). In order to confirm that GI and GV are distinct genotypes, we calculated the mean intra-genotype and inter-genotype distances at the full-length $\mathrm{E}$ gene. As displayed in Table, the mean nucleotide distance between GV and GI

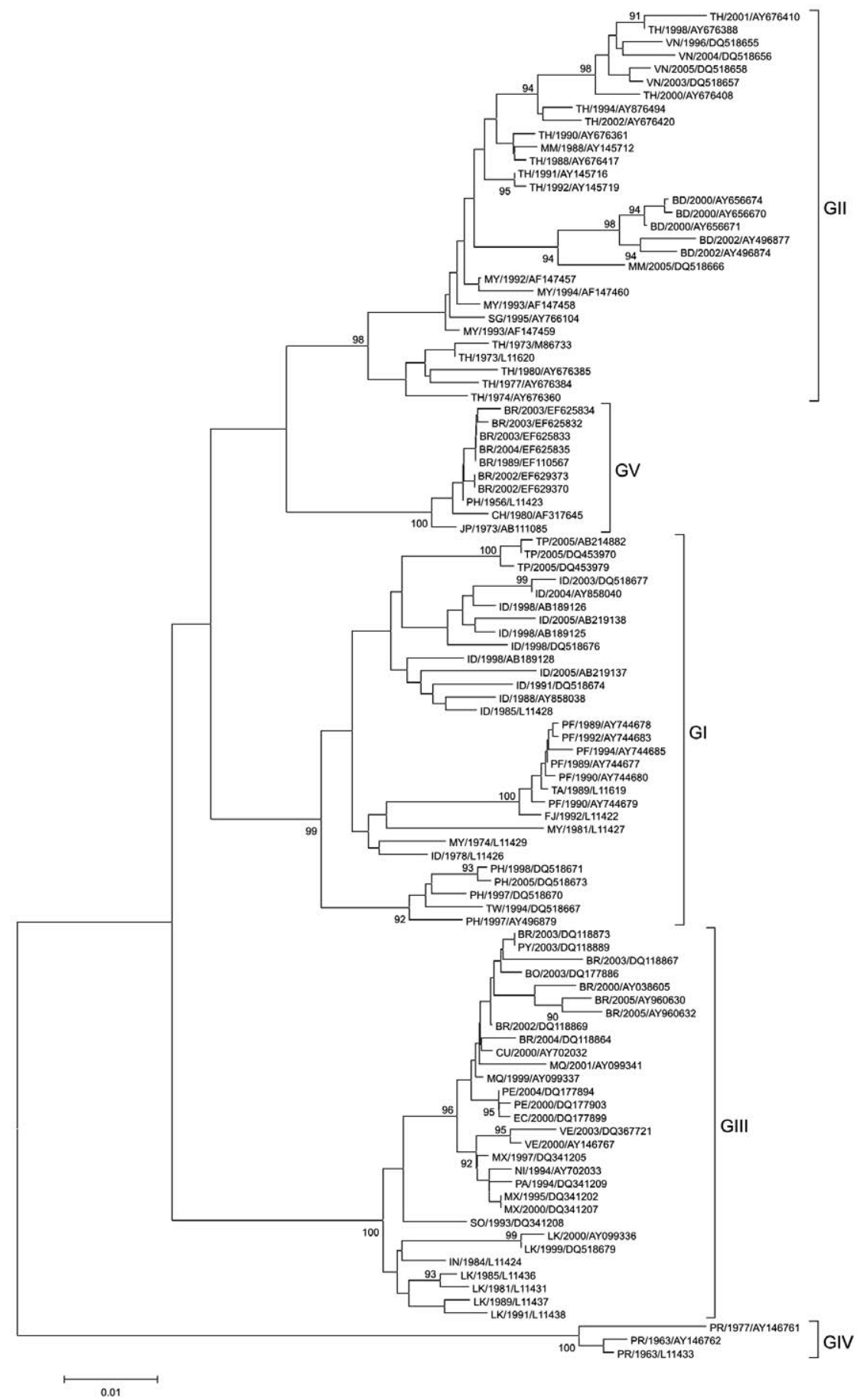

Neighbour-Joining phylogenetic tree of 103 partial envelope gene sequences representing the global diversity of dengue virus (DENV)-3. Genotypes are indicated by brackets. Only bootstrap values $>90 \%$ are shown. The names of DENV-3 isolates include reference to country origin and year of isolation. Horizontal branch lengths are drawn to scale and the tree was rooted using GIV which always appears as the most divergent. Countries represented: BD: Bangladesh; BO: Bolivia; BR: Brazil; CH: China; CU: Cuba; EC: Ecuador; FJ: Fiji; ID: Indonesia; IN: India; JP: Japan; LK: Sri Lanka; MM: Myanmar; MQ: Martinique; MX: Mexico; MY: Malaysia; NI: Nicaragua; PA: Panama; PE: Peru; PF: French Polynesia; PH: Philippines; PR: Puerto Rico; PY: Paraguay; SG: Singapore; SO: Somalia; TA: Tahiti; TH: Thailand; TP: East Timor; TW: Taiwan; VE: Venezuela; VN: Vietnam. 
TABLE

Mean nucleotide distances in envelope gene within (intra-genotype) and among (inter-genotype) dengue viruses-3 genotypes

\begin{tabular}{lrrrrr}
\hline & GI & GII & GIII & GIV & GV \\
\hline GI & $3.0 \pm 0.2^{a}$ & - & - & - & - \\
GII & $7.1 \pm 0.6$ & $2.4 \pm 0.2^{a}$ & - & - & - \\
GIII & $7.5 \pm 0.6$ & $7.6 \pm 0.7$ & $1.8 \pm 0.2^{a}$ & - & - \\
GIV & $11.6 \pm 0.9$ & $11.2 \pm 0.8$ & $11.7 \pm 0.9$ & $1.2 \pm 0.3^{a}$ & - \\
GV & $5.1 \pm 0.5$ & $5.4 \pm 0.6$ & $6.5 \pm 0.7$ & $10.6 \pm 0.9$ & $0.4 \pm 0.1^{a}$ \\
\hline
\end{tabular}

mean intra-genotype $(a)$ and inter-genotype nucleotide distances and \pm standard errors of the mean estimated by the bootstrap method with 100 replicates using MEGA 4 program.

was significantly greater than the mean intra-genotype distance. More importantly, the mean distance from GVGI was not significantly different from the corresponding mean distances from GV-GII or GIII, corroborating the classification of GV as a new genotype distinct from GI.

Phylogenetic analysis of the new DENV-3 sequences isolated in Brazil confirmed their classification as GV, in agreement with Nogueira et al. (2008) (Figure). The clustering of the GV Brazilian and Colombian isolates with old GV strains (Philippines/1956/L11423, Japan/1973/AB11085 and China/1980/AF317645) is fully consistent with the tree topology described by Figueiredo et al. (2008) and Usme-Ciro et al. (2008), but these authors classified the GV strains as GI, based on the initial classification proposed by Lanciotti et al. (1994) that merged these two genotypes into a single group (called GI). The identification of GV in DF/DHF South American samples between 2002-2004 deserves special attention because $\mathrm{GV}$ has been considered an extinct lineage (Araujo et al. 2009) as only three GV strains (from Philippines in 1956, Japan in 1973 and China in 1980) had been found worldwide before the identification of South American GV strains at the turn of the XXI century.

Furthermore, a detailed analysis of the Genbank database, adopting the basic local alignment search tool (www. ncbi.nlm.nih.gov/BLAST), allowed us to identify one additional DENV-3 E sequence (accession EF110567) with a high similarity score to the GV strains, which grouped with the GV genotype in the phylogenetic tree (Figure). Surprisingly, this unpublished sequence was deposited in the Genbank database in 2006, but seems to correspond to a virus isolated in state of Para, Brazil, in 1989 (Bukin et al. 2006). This finding contrast with the official records that DENV-3 was first isolated in Brazil from an autochthonous case in 2000 (Nogueira et al. 2001).

To further explore the genetic relationships between GV strains, we calculated the pair-wise genetic distances among sequences within an overlapping fragment of 822 bp of the E gene. Nucleotide distances between GV Brazilian strains were extremely low, ranging from $0-0.5 \%$. Of note, two GV Brazilian strains isolated near the turn of this century (accession EF625833 and EF625835) were identical to the Brazilian strain from 1989 in the gene fragment analyzed. This analysis further revealed surprisingly low genetic distances $(0.1-0.4 \%)$ between the GV Brazilian isolates and the prototype DENV-3
Philippines/1956/L11423 strain isolated in 1956, which correspond to only 1-3 nucleotide differences throughout the $822 \mathrm{bp}$ fragment. These mean genetic distances were even lower than those between the Philippines/1956/ L11423 isolate and early GV Asian strains [Japan/1973/ AB111085 (0.6\%) and China/1980/AF317645 (0.5\%)] and much lower than those expected from the mean evolutionary rate estimated for the DENV-3 E gene $\left(9 \times 10^{-4}\right.$ substitutions per site per year) (Araujo et al. 2009). No insertions, deletions or point mutations that could distinguish GV Brazilian strains from other strains of this genotype were observed.

The results presented here support the classification of DENV-3 sequences from genotypes I and V as distinct genotypes and confirm that new DENV-3 sequences described in Brazil and Colombia correspond to GV. This study also reveals that a DENV-3 GV sequences, apparently isolated in Brazil in 1989 and deposited in the Genbank database, was identical or very similar in the E gene fragment to the GV Brazilian strains isolated during the 2000s. The GV Brazilian strains were also unexpectedly similar to the prototype DENV-3 strain identified in the Philippines 48 years ago. These results raise some important questions: what is the origin of the GV Brazilian strains? How can GV Brazilian strains isolated during the 2000s display a higher similarity to the prototype Philippines/1956/L11423 isolate than GV Asian strains isolated during 1973 and 1980? More GV sequences from Brazil and elsewhere in South America should be analyzed to answer these questions. Increased surveillance, accurate genetic classification of DENV-3 viruses and molecular epidemiology studies are critical to support new findings and provide an adequate understanding of DENV infections in Brazil.

\section{REFERENCES}

Anonymous 1995. Dengue type 3 infection. Nicaragua and Panama, October-November 1994. Wkly Epidemiol Rec 70: 41-43.

Aquino VH, Anatriello E, Gonçalves PF, DA Silva EV, Vasconcelos PF, Vieira DS, Batista WC, Bobadilla ML, Vazquez C, Moran M, Figueiredo LT 2006. Molecular epidemiology of dengue type 3 virus in Brazil and Paraguay, 2002-2004. Am J Trop Med Hyg 75: 710-715.

Araujo JM, Nogueira RM, Schatzmayr HG, Zanotto PM, Bello G 2009. Phylogeography and evolutionary history of dengue virus type 3. Infect Genet Evol ", in press. 
Balmaseda A, Sandoval E, Pérez L, Gutiérrez CM, Harris E 1999. Application of molecular typing techniques in the 1998 dengue epidemic in Nicaragua. Am J Trop Med Hyg 61: 893-897.

Briseño-García B, Gómez-Dantés H, Argott-Ramírez E, Montesano R, Vázquez-Martínez AL, Ibáñez-Bernal S, Madrigal-Ayala G, Ruíz-Matus C, Flisser A, Tapia-Conyer R 1996. Potential risk for dengue hemorrhagic fever: the isolation of serotype dengue-3 in Mexico. Emerg Infect Dis 2: 133-135.

Bukin EK, Atrasheuskaya AV, Kroon E, Teixeira MM, Ignatyev GM 2006. Dengue epidemic in Para State, Brazil, 1989. Genbank accession number EF110567. Available from: http://www.ncbi.nlm. nih.gov/Genbank/GenbankOverview.html.

Chambers TJ, Hahn CS, Galler R, Rice CM 1990. Flavivirus genome organization, expression and replication. Аnnu Rev Microbiol 44: 649-688.

Figueiredo LB, Cecilio AB, Ferreira GP, Drumond BP, Oliveira JG, Bonjardim CA, Ferreira PC, Kroon EG 2008. Dengue virus 3 genotype 1 associated with dengue fever and dengue hemorrhagic fever, Brazil. Emerg Infect Dis 14: 314-316.

Guzman M, Huelva G, Saenz E, Quiroz E, De los Reyes J, Balmaceda A 1998. Reintroduction del dengue 3 en las Ameritas: 1994-1996. Arch Venez Med Trop 2: 8-19.

Istúriz RE, Gubler DJ, Brea del Castillo J 2000. Dengue and dengue hemorrhagic fever in Latin America and the Caribbean. Infect Dis Clin North Am 14: 121-140.

Lanciotti RS, Lewis JG, Gubler DJ, Trent DW 1994. Molecular evolution and epidemiology of dengue-3 viruses. J Gen Virol 75: 65-75.
Lindebach BD, Rice CM 2001. Flaviviridae: the viruses and their replication. In DM Knipe, PM Howley (eds.), Fields Virology, 4th ed., Lippincott Williams and Wilkins, Philadelphia, p. 991-1041.

Nogueira MB, Stella V, Bordignon J, Batista WC, Borba L, Silva LH, Hoffmann FG, Probst CM, Santos CN 2008. Evidence for the cocirculation of dengue virus type 3 genotypes III and V in the Northern region of Brazil during the 2002-2004 epidemics. Mem Inst Oswaldo Cruz 103: 483-488.

Nogueira RM, Miagostovich MP, de Filippis AM, Pereira MA, Schatzmayr HG 2001. Dengue virus type 3 in Rio de Janeiro, Brazil. Mem Inst Oswaldo Cruz 96: 925-926.

Rosen L 1999. Comments on the epidemiology, patogénesis and control of dengue. Med Trop 59: 495-498.

Tamura K, Dudley J, Nei M, Kumar S 2007. MEGA4: Molecular Evolutionary Genetics Analysis (MEGA) software version 4.0. Mol Biol Evol 24: 1596-1599.

Usme-Ciro JA, Mendez JA, Tenorio A, Rey GJ, Domingo C, GallegoGomez JC 2008. Simultaneous circulation of genotypes I and III of dengue virus 3 in Colombia. Virol J 5: 101.

Usuku S, Castillo L, Sugimoto C, Noguchi Y, Yogo Y, Kobayashi N 2001. Phylogenetic analysis of dengue-3 viruses prevalent in Guatemala during 1996-1998. Arch Virol 146: 1381-1390.

Uzcategui NY, Comach G, Camacho D, Salcedo M, Cabello de Quintana M, Jimenez M, Sierra G, Uzcategui RC, James WS, Turner S, Colmes EC, Gould EA 2003. Molecular epidemiology of dengue virus type 3 in Venezuela. J Gen Virol 84: 1569-1575. 